\title{
The Structure of Monitoring Node and Monitoring Center of Environmental MonitoringSystem
}

\author{
Yijie $\mathrm{Li}^{1}$ \\ ${ }^{1}$ Guangdong Peizheng College, Guangzhou, China
}

\begin{abstract}
The monitoring items shall be determined in a scientific and reasonable way according to the environmental monitoring standards and specifications, The purpose of environmental monitoring is to reflect the monitoring data of environmental monitoring system in real time, accurately and comprehensively. It provides scientific basis for environmental planning and macro decision-making.It makes the environmental monitoring system feasible and economical. Guided by the technical route of environmental monitoring, combined with the practical principle and priority monitoring principle, the comprehensive planning and reasonable arrangement are made. Environmental protection, scientific research and other purposes. Aiming at the monitoring node and monitoring center of environmental monitoring system, this paper analyzes the important position of environmental management and monitoring plan. This paper discusses the composition and structure of the monitoring system, in order to maximize the role of environmental management, reduce and mitigate the impact of monitoring projects on the ecological environment, and realize the sustainable development and operation of environmental system monitoring.
\end{abstract}

\section{Main purpose of environmental monitoring}

The purpose of environmental monitoring is to reflect the development trend of environmental monitoring system in real time, accurately and comprehensively, and to provide scientific basis for environmental planning and management, environmental impact and assessment, pollution control and macro decision-making.

Facing the serious challenge of environmental problems, we must take a series of measures to protect and improve the environment. In order to find out the causes of environmental quality changes, the nature, source, content and distribution of pollutants were investigated, with emphasis on qualitative and quantitative analysis of environmental pollutants. The main object of environmental monitoring is all kinds of pollutants discharged in the environment due to human production activities. Most of the analysis is carried out in the laboratory after sampling. Environmental analysis characterized by discontinuous operation can only analyze and determine local, short-term and single pollutants.

Environmental monitoring is the use of chemistry, physics, biology, medicine, remote sensing, remote sensing, computer and other modern scientific and technological means to monitor, determine, monitor and reflect the environmental quality and its changing trend of various indicators of data, so as to make a comprehensive evaluation of environmental quality. Environmental monitoring includes the monitoring of chemical pollutants and the monitoring of physical factors such as noise, vibration, heat, electromagnetic radiation and radioactivity. It also includes the biological monitoring of all kinds of reactions and information tests sent out by organisms due to changes in environmental quality, as well as the ecological monitoring of regional community and population migration and changes. Environmental analysis and environmental monitoring are two important concepts which are closely related and different. Environmental monitoring is developed on the basis of environmental analysis. Compared with environmental analysis, environmental monitoring includes a wider range, more complex objects, more profound contents, and more comprehensive means and methods.

Environmental monitoring is to determine the environmental quality and its change trend by determining the representative values of the factors affecting the environmental quality. The process of environmental testing includes accepting tasks, on-site investigation and data collection, monitoring plan design, optimizing distribution, sample collection, sample transportation and preservation, sample pretreatment, analysis and testing, data processing, comprehensive evaluation, etc. The comprehensive nature of environmental monitoring, monitoring means include chemistry, physics, biology, physical chemistry, biochemistry, biophysics and other methods that can characterize the environmental quality. Monitoring objects include air, water, soil, solid waste, biological and other objects. In the process of statistical processing and comprehensive analysis of monitoring data, the natural and social conditions of the area must be considered comprehensively. Objects of environmental 
monitoring: natural factors, human factors, pollution components. Environmental monitoring includes chemical monitoring, physical monitoring, biological monitoring and ecological monitoring

The purpose of environmental monitoring is to accurately, timely and comprehensively reflect the current situation and development trend of environmental quality, and provide scientific basis for environmental management, pollution source control and environmental planning. It can be summarized as follows: To evaluate the environmental quality according to the environmental quality standards; to track and find the pollution sources according to the pollution distribution, so as to provide the basis for the supervision and control of pollution; to collect background data and accumulate long-term monitoring data, so as to provide data for the research of environmental capacity, the implementation of total amount control and target management, and the prediction of environmental quality; to protect human health and the environment To make rational use of natural resources, formulate environmental regulations, standards, planning and other services.

\section{Main technical indexes of environmental monitoring}

The technical route of environmental monitoring determines the development direction of environmental monitoring, restricts the selection and application of monitoring technology, and affects the formulation and implementation of monitoring scheme. China's environmental monitoring technology route currently puts forward nine monitoring technology routes in terms of air, surface water, noise, pollution sources, ecology, solid waste, soil, biology and environmental radiation, which is a systematic study and comprehensive summary of China's environmental monitoring work. It has both similarities and differences in its route and elements, and considers the unification of environmental requirements At the same time, it gives consideration to the stage of regional development. It not only focuses on the current actual needs, but also forecasts the long-term development in the future. This paper not only expounds the scientificity of the technical route, but also demonstrates the feasibility of implementing the technical route. It is a comprehensive and feasible technical route for environmental monitoring in China at present.

The monitoring range of air pollution should be divided according to the air quality, topography and underlying surface finish, meteorological factors, pollutant emission height and quantity, environmental function area and other factors. The monitoring range of water body can be determined according to different water body conditions. The monitoring scope of surface water must include the areas with obvious impact on water environment, which should fully reflect the basic environmental conditions related to surface water and fully meet the requirements of monitoring purposes. The determination of groundwater monitoring scope should consider the environmental hydrogeological conditions, groundwater exploitation, pollutant distribution and diffusion forms, and regional hydrochemical characteristics. Generally, the waste water pollution source is discharged through pipelines and ditches with small cross-sectional area, so it is not necessary to set a cross-section to determine the sampling location directly.

\section{Environmental monitoring system node and monitoring center}

The technical route of environmental monitoring refers to the technical means and ways adopted to complete the given monitoring task and achieve the monitoring purpose in a certain period of time. Monitoring system stability and variability, stability refers to a certain period of time, once the technical route is determined, to maintain its relative stability. Variability refers to the need of environmental management and the development of monitoring technology, the technical route needs to be adjusted and improved regularly or irregularly, and the diversity of technical means and approaches.

The environmental monitoring system is composed of monitoring node, collection node and monitoring center.

The environmental monitoring node samples the air, surface water and noise information to the node, which is simply processed and stored by MCU. It can do the necessary output and display, and transmit the information to the collection node through the communication module

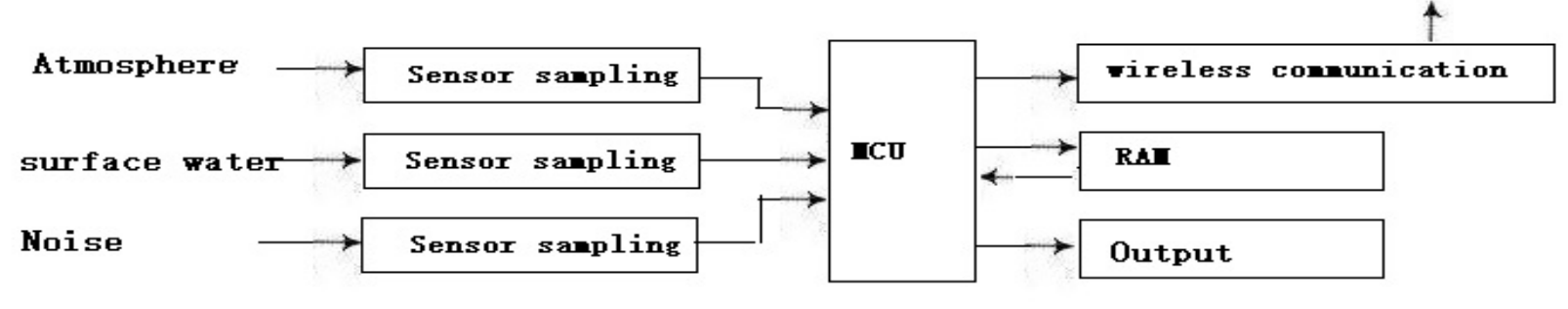

Figure 1 composition diagram of monitoring node MA

MB environmental monitoring node

The environmental monitoring node samples the soil, ecological and biological information to the node, which is simply processed and stored by MCU. It can do the necessary output and display, and transmit the information to the collection node through the communication module. 


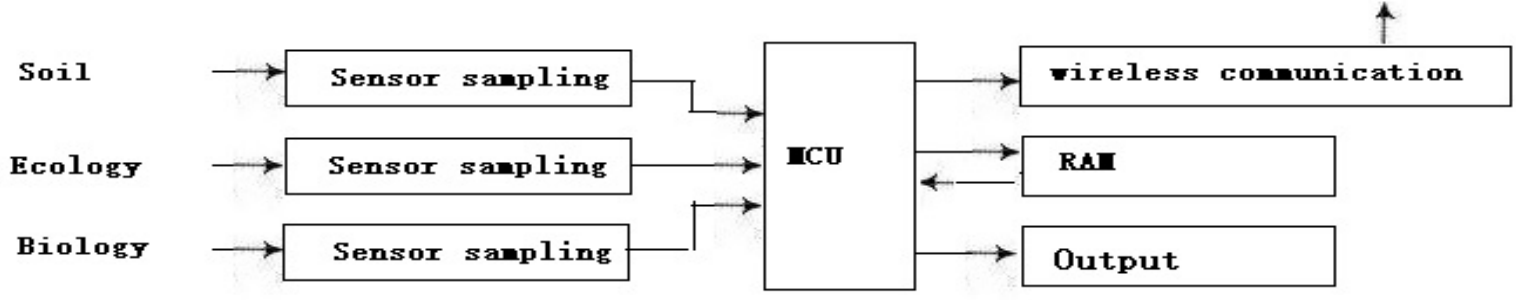

Figure 2 composition diagram of monitoring node MB

MC environmental monitoring node

The environmental monitoring node samples the information of pollution source, solid waste and radiation source to the node, which is simply processed and stored by MCU. It can do the necessary output and display, and transmit the information to the collection node through the communication module.

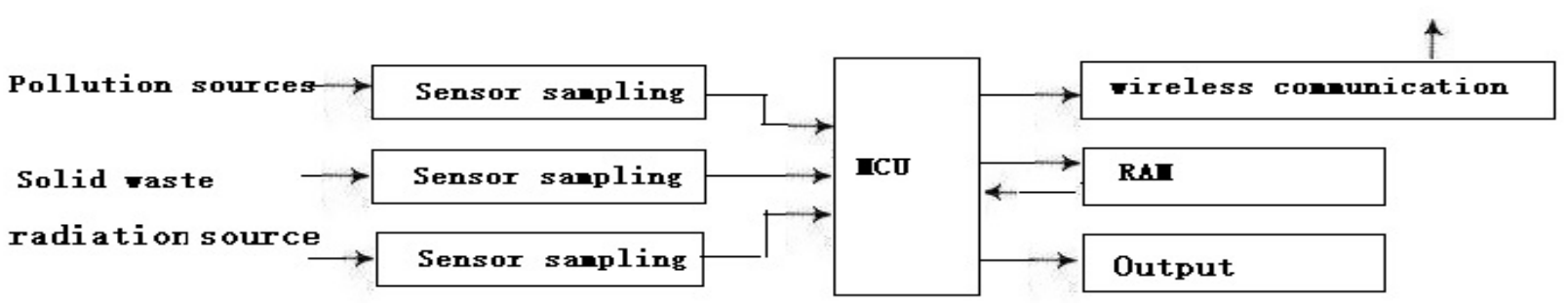

Figure 3 composition diagram of monitoring node $\mathrm{MC}$

Environmental monitoring center and monitoring node

The environmental monitoring center receives the information of MA / MB and MC from the environmental monitoring collection point, and then processes and analyzes the environmental information.

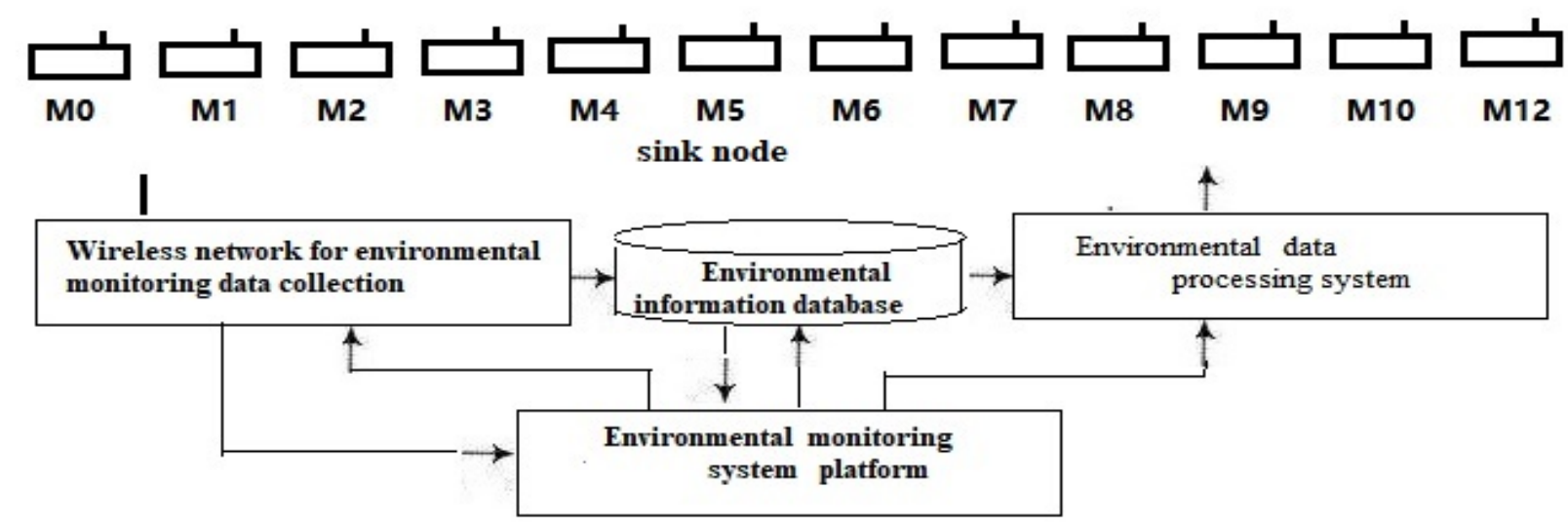

Figure4 environmental monitoring center and monitoring nodes

The monitoring center is composed of wireless network communication system, environmental data processing system, environmental information database and monitoring system platform. The wireless communication system receives the monitoring data from the collection node and stores it in the database. The environmental data processing system processes and analyzes the monitoring data. The monitoring system control platform completes the control, scheduling and coordination of the monitoring system to ensure the coordinated and orderly work of the monitoring system.

\section{Conclusion}

This paper discusses the main technical indicators of environmental monitoring system monitoring, discusses the composition and principle of environmental monitoring node, and the system architecture of environmental monitoring center. To provide technical guarantee for the establishment of environmental monitoring system. Environmental monitoring is developed along with the emergence of environmental pollution. The automatic monitoring system and ecological monitoring system have been established. With the help of computer technology, network technology and Internet of things technology, geographic information system, remote sensing technology and global satellite positioning system, we can continuously observe the changes of air and water pollution and ecological environment, predict and forecast the future 
environmental quality, which is of great significance It has greatly expanded the scope of environmental monitoring and the ability of acquisition, processing, transmission and application of monitoring data, provided strong technical support for dynamic monitoring of regional environmental quality and ecological environmental quality, and greatly promoted the modernization of environmental monitoring.

\section{reference}

1. Hu Hui. Discussion on how to build an advanced environmental monitoring and early warning system for municipal and county environmental monitoring departments, 2012, Chinese society of Environmental Sciences, 2012

2. Zhang Yu. Suggestions on radiation environmental monitoring in China in the post Fukushima era, Symposium on nuclear and radiation safety supervision, 2013

3. Su Mei Wen. The development status of Internet of things and the strategic choice of its development mode in China [J]. Research on technology, economy and management, 2015

4. Wang Yi. Research on smart home based on wireless sensor of Internet of things [J]. Computer technology and development, 2015

5. Wang Jian. Discussion on rural environmental monitoring technology. The Third Symposium on heavy metal pollution prevention and risk assessment and Professional Committee on heavy metal pollution prevention and control, 2013

6. Lu Guibin. discussion on environmental monitoring information disclosure, Chinese society of Environmental Sciences, 2013

7. Chen Jing. Discussion on file awareness of environmental monitoring workers, Chinese society of Environmental Sciences, 2012 